\title{
Axon/myelin traffic of Theiler's virus during persistent CNS infection
}

\author{
Jean-Pierre Roussarie ${ }^{1}$, Claude Ruffie${ }^{1}$, Julia M Edgar ${ }^{2}$, Ian Griffiths ${ }^{2}$ and \\ Michel Brahic*1,3
}

\author{
Address: ${ }^{1}$ Department of Virology, Institut Pasteur, Paris, 75724 Cedex 15, France, ${ }^{2}$ Institute of Comparative Medicine, University of Glasgow, \\ Glasgow, UK and ${ }^{3}$ Department of Microbiology-Immunology, Stanford University, Stanford CA 94305, USA \\ Email: Michel Brahic* - mbrahic@stanford.edu \\ * Corresponding author
}

from Infectious diseases of the nervous system: pathogenesis and worldwide impact Paris, France. 10-13 September 2008

Published: 23 September 2008

BMC Proceedings 2008, 2(Suppl I):S38

This abstract is available from: http://www.biomedcentral.com/I753-656I/2/SI/S38

(c) 2008 Roussarie et al; licensee BioMed Central Ltd.

Theiler's virus, a murine picornavirus, persists for life in the central nervous system (CNS) of mouse and causes a demyelinating disease, which is a model for multiple sclerosis. The virus infects neurons first but persists in white matter glial cells, mainly in oligodendrocytes and macrophages. The mechanism by which the virus traffics from neurons to glial cells and the respectives roles of oligodendrocytes and macrophages in persistence are poorly understood.

We took advantage of our previous finding that the shiverer mouse, a mutant with a deletion in the myelin basic protein gene, is resistant to persistent infection to examine the role of myelin in persistence. Using immune chimeras, we showed that resistance is not mediated by immune responses or by an efficient recruitment of inflammatory cells into the CNS. With both in vivo and in vitro experiments, we showed that the mutation does not impair the permissiveness of neurons, oligodendrocytes and macrophages to the virus. We demonstrated that viral antigens are present in cytoplasmic channels of myelin during persistent infection of wild type mice. Using retinal ganglion neurons and the optic nerve as a model, we showed that the virus traffics from the axons of retinal ganglion cells into the cytoplasmic channels of myelin and that this traffic is impaired by the shiverer mutation. Importantly, this traffic takes place in the absence of axonal degeneration, as shown using the Wlds mutant mouse.
These results show that axon to myelin traffic and the infection of myelin/oligodendrocytes are essential steps in the establishment of a persistent CNS infection by Theiler's virus. They uncover an unsuspected axon to myelin traffic of a non-enveloped viral particle. We hypothesize that the virus insinuates itself into a pre-existing pathway of transport of axolemma material into the surrounding myelin. The existence of such a pathway has been suggested in the past. It could be an important part of the supporting role played by myelin in the physiology of the axon. 